\title{
Negocios y género: el hombre como sexo débil y propuestas educativas \\ (Business and gender: the man as a weak sex and educational proposals)
}

\section{'Andrés Ramírez Puerto}

\begin{abstract}
We can't deal with the ethical and economic consequences of the gender issues unless we are aware of this ideology. There is evidence of an outstanding performance of women in business what has been linked with a flexible femininity together with its attitudes and values demanded in today's economy. Men's studies on their part have shown a rigid masculinity that prevents many men from adapting to the business environment. This article reflects about the internalization of this ideology that shows resistance to media campaigns so we need to offer a specific gender training for managers and include gender education in schools. We need to go down to the level of the values and narratives internalized, encouraging reflexion, to expand our views and capabilities.
\end{abstract}

Key words: business, crisis, education, gender, masculinity.

JEL: A14, B54.

Resumen: Un insuficiente reconocimiento de la influencia de la ideología de género nos impide prevenir consecuencias negativas éticas y económicas. En el terreno del liderazgo existe evidencia de un mayor éxito de las mujeres empresarias que ha sido relacionado con una mayor flexibilidad de las mujeres, con actitudes y valores femeninos demandadas por la economía actual. Por su parte, los estudios sobre masculinidad muestran una estructura rígida y excluyente que lleva a la inadaptación de muchos hombres. Este artículo reflexiona sobre la naturalización, interiorización y resistencia ideológicas, planteando ir más allá de campañas mediáticas para la concienciación, necesitándose formación específica en género tanto a nivel empresarial como universitario y escolar. Necesitamos descender al nivel de los valores y las narrativas interiorizadas, fomentando la reflexión, para expandir la capacidad de visión y de acción.

Palabras clave: crisis, educación, empresa, género, masculinidad. 


\section{Introducción}

Los conocimientos populares sobre el género siguen estando reducidos a sus significados locales sin llegar al nivel de una suficiente reflexión sobre su cualidad construida, sus mecanismos, valores y narrativas. Esta inconsciencia sobre una identidad social tan estructuradora impide regular su influencia y los límites que impone a la hora de combatir las desigualdades. Aparte de las implicaciones éticas se han ido haciendo patentes otras limitaciones que ejerce la identidad de género y particularmente la masculinidad, en cuanto un deficiente rendimiento estudiantil, laboral y, tal y como se va a analizar el presente artículo, el éxito empresarial.

Existe evidencia que relaciona a las líderes empresariales con un mayor éxito relativo, a pesar de encontrarse las mujeres en minoría en esta actividad. Paralelamente, la relación entre hombres empresarios y su menor rendimiento en los tiempos actuales, rápidamente cambiantes, se ha relacionado con una masculinidad problemática, que limita por su rigidez ideológica, tanto la capacidad de adaptación al entorno de negocios del propio empresario, como el ascenso de mujeres competitivas al establecer el techo de cristal (Carr-Ruffino, 1991), perjudicando en ambos casos el éxito empresarial y económico.

En los siguientes apartados voy a exponer datos producidos tanto desde organizaciones para el análisis económico como desde la prensa especializada y la academia. Veremos cómo existe una correlación positiva entre la presencia de mujeres directivas y rendimiento empresarial. A continuación citando estudios al respecto, reflexionaremos sobre el papel de la ideología de género en estas diferencias bajando al nivel de los valores asociados con la feminidad y la masculinidad, de la rigidez en la estructura, la narrativa y el nivel de conciencia de cada una de estas dos identidades.

Esto nos llevará a considerar si las acciones correctoras que se plantean en la actualidad para la mejora de la economía y la sociedad en base a aprovechar todo el potencial humano (eliminando desigualdades improductivas) van dirigidas en el sentido correcto. Teniendo en cuenta que las acciones mediáticas puntuales (desde nivel estatal en España y países latinoamericanos) sobre concienciación de género no abarcan toda su complejidad ni resultan suficientes para lograr los cambios necesarios, debemos replantear la estrategia.

Argumentaré sobre la necesidad de una educación sobre género que lleve a la reflexión tras la concienciación, en el sentido de sacar a la superficie 
lo interiorizado, inconsciente para el individuo pero conocido por los científicos sociales; la reflexión sobre las limitaciones que nos impone y las consecuencias en el entorno empresarial en particular, en pro de una mejora económica, y en el entorno social más general. Sin embargo, una actuación formativa en este ámbito, dirigida a profesionales, aunque de importancia clave, debe ser acompañada de educación en género para la sociedad más amplia, a través de su inclusión en la currícula de etapas educativas obligatorias, tal y como defienden numerosos científicos sociales, abordando así de raíz problemáticas tan importantes como la violencia de género y la desigualdad general.

\section{Conciencia de género}

La identidad de género al igual que las demás identidades sociales, es parte importante de la identidad de cada persona estableciendo valores, narrativa, lógicas que guían nuestro pensamiento y comportamiento, nos sitúa ante los demás y funciona como marco interpretativo en la comprensión y comunicación social (Giménez, 2009; Ramírez, 2007).

Somos conscientes de lo que significa y conlleva ser hombre o mujer en nuestra sociedad, es decir los valores y comportamientos atribuidos en función del sexo, sin embargo generalmente ignoramos el hecho de que se trata de una ideología atribuida, es decir, con la cualidad de ser construida y particular. Los mecanismos de esta ideología, sus narrativas internas, su influencia en nuestro pensamiento y acción cotidianos pasan desapercibidos. Así esta inconciencia acaba teniendo consecuencias en las relaciones con los demás en los diversos entornos sociales: familia, trabajo, estudios... llegándose incluso al nivel de racionalmente creer en la igualdad pero tener un comportamiento que la perpetúa (Colás y Villaciervos, 2007).

Los estudios sociales de género tienen una larga tradición de análisis y reflexión sobre estos mecanismos, lógicas y narrativas que condicionan el comportamiento social. Durante décadas se ha estudiado especialmente la situación de la mujer por su situación de exclusión en diferentes ámbitos sociales, atribuyéndose esto a una ideología de género que perjudicaba el desarrollo de su potencial. Sin embargo, en los últimos años se han realizado muchos estudios sobre la masculinidad como identidad problemática, no ya sólo con respecto a las mujeres y la distribución de poder, sino también para los mismos hombres, al actuar como un sistema de valores y lógicas interiorizado que limita la visión 
y la capacidad de respuesta ante un mundo en constante cambio; generando en muchos hombres conflictos internos, como la incompatibilidad de emociones e ideologías interiorizadas con la presión social y lógica de tener que reconocer la ética de la igualdad.

Uno de los entornos de interacción social en los cuales encontramos muestras de una masculinidad problemática es el entorno empresarial, particularmente en su liderazgo, una posición social hasta hace poco casi exclusivamente masculina y que hoy con una presencia aun minoritaria pero creciente de mujeres, deja patente hechos distintivos atribuibles al género. El sistema de valores y la actitud flexible de la feminidad se asocia con una ampliación de la visión y la capacidad de actuación que coloca a los hombres en relativamente inadaptados al entorno económico cambiante y cada vez más complejo. Analicemos las limitaciones masculinas en el liderazgo.

Desde los estudios de género se ha definido la masculinidad hegemónica (Valcuende y Blanco, 2015; Kimmel, 1997) como una identidad social rígida y excluyente, siendo por tanto su contraparte, la feminidad, más flexible, cualidad prerrequisito para el cambio y la adaptación al entorno, fundamental en el ámbito de la dirección empresarial.

Existen estudios que señalan una especial interiorización de la ideología de género en los hombres, (Colás y Villaciervos, 2007; Amurrio, et al, 2012) algo relevante en esta cuestión en cuanto a que la falta de consciencia de los hombres sobre la ideología de género, hará que su influencia en el comportamiento resulte en gran medida desapercibida, impidiendo la reflexión sobre ello y la libertad de actuación. Siendo el sistema de género tradicional, es decir pre-moderno y por tanto inadaptado a unos tiempos en los que se debate la superación de la misma modernidad.

La falta de coherencia entre lo aceptado racionalmente por muchos hombres y su comportamiento señala igualmente la inconsciencia de esta dimensión en el día a día, con consecuencias problemáticas para los propios hombres y para la sociedad en general, que hace que ni leyes o reglas sociales ni las campañas mediáticas por la igualdad sean suficientes para lograr los cambios deseados.

La insuficiente reflexión sobre las limitaciones ideológicas del constructo de género ha dado lugar a propuestas para incluir formación específica identitaria de género en los sistemas educativos, como materia transversal, sin embargo no ha pasado a ser en el mejor de los casos una enseñanza puntual dentro de una asignatura general, no obstante hay que reconocer que la educación sexual 
si se ha abierto paso en cada vez más sistemas educativos, buscando logros concretos en materia de enfermedades de transmisión sexual y embarazos no deseados. Es necesaria por tanto una mayor formación en género que aborde las identidades en profundidad, tanto en ambientes y sectores concretos como los empresarios y la masculinidad problemática, como sobre hombres y mujeres en general, como estrategia para desenmascarar las identidades sociales interiorizadas, fomentando la reflexión y la libertad individual.

Veamos a continuación la relación del género con el éxito empresarial a través de las empresarias de éxito que contrastan con hombres que muestran no tener la flexibilidad necesaria para la adaptación a los constantes cambios en el entorno.

\section{Empresarias de éxito en un entorno masculino}

El alto rendimiento relativo de las mujeres empresarias fue analizado en un primer momento desde la literatura feminista. Helgesen (1990) y Rosener (1995) señalaron que estas mujeres aun estando en un entorno en el que se encuentran en minoría, en una subcultura marcada por la masculinidad, muestran una especial efectividad en su actuación.

Desde el lado de la prensa económica y de negocios, a primeros de nuestro siglo, la cuestión del éxito de las mujeres en el mundo de los negocios pasa a ser un tema recurrente. La revista Business Week (Conlin, M) publica en el año 2003: "la nueva división de género: los hombres podrían resultar los perdedores en una economía global que valora más el poder mental que el poderío anterior". The Boston Globe (Tuhus-Dubrow, R) titula un artículo en el año 2009: "La ventaja femenina. Una nueva razón para fomentar las mujeres en los negocios: es más rentable", en el cual se revisan los resultados de diversas investigaciones al respecto.

John Demartini, gurú de los negocios señala como valores para el triunfo "la comunicación (con el equipo y los clientes), la actitud de servicio, respeto y tolerancia, la empatía, escucha, diálogo, el trabajo en equipo, la motivación en lugar del mando y ordeno, la expansión de la visión", valores como vemos, en la órbita de los tradicionalmente femeninos.

Mercedes García (2009) enumera las características de las mujeres directivas, entre las que recoge la inclinación a la cooperación, empatía, la flexibilidad y la creatividad, afirmando que estas características femeninas hacen que la gestión empresarial sea más efectiva y mejor adaptada a un ambiente como el actual 
(complejo, cambiante y multidimensional), que depende en gran manera de la capacidad para relacionarse y comunicar.

Estudios de la empresa de trabajo temporal Manpower España y Small Business Administration de México publicados en la revista mexicana Alto Nivel, concluyen que la mayoría de las empresarias, a diferencia de los hombres no tienen como primera motivación el éxito económico sino la autorrealización. Entre los factores de éxito femenino resaltan: "la intuición, la reflexión-análisis y la prudencia". Las mujeres empresarias describen sus empresas en términos familiares y los negocios como "red de contactos", dando un toque personal que también motiva a sus propios empleados.

Women matter 2, una extensa investigación internacional realizada por Mckinsey \& Company sobre 9 actitudes clave para el éxito empresarial, dio como resultado que las mujeres líderes sobresalían claramente en cuatro de ellas: estimulación intelectual, inspiración, proceso de decisión participativo, expectativas y premios; habiendo entre las actitudes restantes poca variabilidad por sexos.

En marzo del 2015 la revista Fortune publica el estudio del instituto Quantopian, que realiza una comparación entre 80 mujeres CEO (gerentes) del listado Fortune 1000 (las mayores compañías del mundo), comparando las rentabilidades de sus empresas con la media de las compañías que recoge el índice bursátil S\&P500. Los resultados son los siguientes:

Los media de los beneficios de las compañías dirigidas por mujeres son un $226 \%$ superiores a la media del S\&P500

Otro estudio realizado por la organización Catalyst Research durante cuatro años, en las que se compara el rendimiento empresarial en base a la evolución del dinero invertido en éstas, compara las empresas que recoge el índice Fortune 500 (las 500 mayores del mundo) distribuyéndolas en cuanto al número de participantes mujeres en sus consejos de administración, y oponiendo los resultados de las que se encuentran en el primer cuartil (3 o más mujeres) con el último cuartil, concluyendo que, las compañías con más directivas se sitúan por delante de la siguiente manera:

El valor del capital neto aumenta un $53 \%$ con respecto al $4^{\circ}$ cuartil, los resultados en ventas son el $42 \%$ superior y el capital invertido se revalorizó un 66 $\%$ más. 


\section{Género y liderazgo desde la academia}

Muchos de estos trabajos sobre el éxito relativo de las mujeres hacen referencia explícita sobre los valores que demanda la economía actual, como el servicio y la satisfacción del consumidor o la gestión de los recursos humanos, y su correspondencia con los de la feminidad tradicional.

Desde el mundo académico esta cuestión no ha pasado desapercibida, Adams y Ferreira $(2007,2009)$ tras analizar los liderazgos empresariales por sexos afirmaron que existe una clara evidencia de que las mujeres tienden a mostrar más aversión al riesgo que los hombres, llevando a relacionar una mayor participación femenina con menores probabilidades de sufrir crisis económicas. Gaddis y Dezso (2012) observaron que la organización interna se ve reforzada existen mujeres directivas, quienes logran mayor implicación de los otros altos directivos y de los mandos intermedios.

La relación entre la diversidad de sexos en la dirección de la empresa y un mayor rendimiento está respaldada por múltiples estudios, en cambio el análisis sobre cuál sería la relación óptima entre sexos no arrojó resultados concluyentes, según Eagly \& Carli $(2003,2007)$, quienes reclaman más atención sobre el fenómeno del techo de cristal como limitante del éxito empresarial. En su trabajo incluyen la siguiente observación:

Existe una gran dificultad para el análisis del rendimiento femenino debido a la que cualquier ventaja de éstas podría quedar bloqueada por desventajas que surgen de un entorno en el que se dan prejuicios y discriminación hacia las mujeres líderes. (Eagly \& Carli, 2003)

Otras conclusiones de los trabajos de Eagly \& Carli (ib.) son, por un lado, la constatación del hecho de que las organizaciones cambian, insertas en contextos competitivos, cambios de mercado, cambios sociales y culturales, y que las mujeres cambian:

Las mujeres cambian, asumen las características personales requeridas para triunfar en los nuevos roles, en entornos masculinos. Las mujeres se vuelven más masculinas pero sin decrecer en cuanto a cualidades femeninas. 


\section{Feminidad, masculinidad y naturalización}

Hemos visto cómo diversos autores concluyen que existe una especial capacidad de cambio y adaptación de las mujeres, algo patente a través de su particular eficacia en el entorno cambiante empresarial, el cual exige actitudes de escucha, atención al cliente y satisfacción de sus necesidades, el cuidado de las relaciones en la organización, entre otras actitudes tradicionalmente de la órbita femenina. Valores relacionados con la masculinidad y el mundo empresarial como la competencia o el dominio de mercado, aunque necesarios resultan insuficientes en un contexto más complejo que el que existía en la primera modernidad o sociedad de productores (Bauman, 2007), claramente las y los individuos empresarios necesitan ampliar sus roles y acciones incluyendo aspectos antes considerados masculinos y femeninos. Pero como vamos viendo son las mujeres quienes por lo general muestran más flexibilidad y capacidad de adaptación.

Las mujeres, tradicionalmente han convivido con una ideología de género que les otorgaba el papel de delicadas, serviciales y una mayor debilidad, mientras que han tenido que realizar trabajos duros, mostrando fortaleza y resistencia en su vida práctica. Esto nos lleva a la siguiente cuestión, la de una falta de ajuste entre las ideología social y los hechos, y con ello una vivencia desigual de la identidad de género.

Colás y Villaciervos (2007) señalan una especial interiorización de los estereotipos de género entre los hombres jóvenes. Amurrio, et al, (2012) señalan la dificultad de concienciación social sobre la masculinidad y su relación con un fuerte arraigo a nivel inconsciente, desde donde puede generar una influencia en el pensamiento y comportamiento sin pasar por la conciencia y la reflexión.

Esta naturalización resulta paradójica desde los estudios desde los estudios de género, que descubrieron ya tempranamente (Ortner, 1974), la relación ideológica entre mujeres y feminidad como natural, asociándose la masculinidad con la cultura (cualidad construída). La feminidad se ha visto culturalmente como algo intrínseco a la mujer, cercano a lo biológico, inevitable; la masculinidad algo que se tenía que demostrar, probar, reforzar, defender, una identidad que se podía perder si no se vigilaba la conducta y se actuaba erróneamente (como mujer, niño u homosexual). Sin embargo no existe contradicción si pasamos de una perspectiva social a otra personal. Una cosa sería el discurso social que otorga significación al género y otra el grado de interiorización de éste en el propio individuo, hombre o mujer. 
Actuar de una forma no reflexiva ni consciente de los valores que nos mueven, supone vernos limitados por una ideología que, interiorizada y sin ser objeto de reflexión, nos está definiendo la visión y limitando nuestra capacidad de actuación. El mundo real de las mujeres superaba tradicionalmente al sistema de valores que tenían asignado, tal y como he comentado, en cambio, la oficial condición de la masculinidad como construida ha podido ser vivida en cambio como identidad esencial. Es posible que el hombre, para defender ideológicamente un sistema que le proveía de privilegios, debiera de considerar la masculinidad como algo legítimo, real, necesario, lo cual podría haber contribuido a su "naturalización".

Está bien documentada desde los estudios de género la narrativa subyacente en la masculinidad, la rígida oposición de sus roles y valores a lo no masculino, y la presión que crea el mismo entorno de hombres en el comportamiento de género, como vamos a ver a continuación.

\section{Masculinidad, rigidez e inconsciencia}

Valcuende y Blanco (2015) nos ofrecen una minuciosa descripción de esta identidad, en realidad plural: masculinidades, que no obstante mantienen a la masculinidad hegemónica como referente en diversa medida. La masculinidad hegemónica tal y como la describió Kimmel (1997) se fundamenta en el poder, partiendo de un sistema de género dicotómico y jerárquico que excluye tanto a mujeres como gran parte de los hombres. Gestionar el poder implica:

....autocontrol (...) suprimir toda una gama de emociones, necesidades y posibilidades, tales como el placer de cuidar a los otros, la empatía y compasión (Kaufman, 1995:150)

Aparece aquí una clara limitación masculina en cuanto a las necesidades empresariales de crear y promocionar productos sirviendo a los consumidores 0 una adecuada gestión de los recursos humanos. La masculinidad hegemónica, instituida en forma negativa (en oposición a: mujer, niño...) muestra una dependencia de la otredad que no ocurre en el caso femenino. Según Guasch (2003: 122), el individualismo (actual) disuelve la noción de identidad (tal y como se conocía), pero, 
...la masculinidad tradicional continúa existiendo porque en un momento de cambios sociales rápidos ofrece a los varones auto-conceptuación y socialización masculina que fortalece la autoestima por pertenencia grupal.

Blanco (2012), nos indica que no deja de ser paradójico que para un hombre, el seguir el modelo de éxito social de la masculinidad...

...conlleve habitualmente al fracaso personal, cuando no a la exclusión por el ejercicio de la autosuficiencia, cuando no de violencia, o el escaso desarrollo de elementos femeninos como el cuidado.

Pero por sí solo, un sistema ideológico de valores y roles no debería ser un obstáculo para su adaptación al entorno si no estuviese acompañado por su interiorización y su localización en el inconsciente, ocultando sus mecanismos y su influencia en la visión y comportamiento. Es necesario por ello un trabajo sistemático de concienciación a través de la educación específica en género, dirigido tanto a líderes como al conjunto de la sociedad que permita la reflexión sobre los valores y lógicas que nos mueven.

\section{Educación en género para el desarrollo económico y social}

Estamos reflexionando sobre la influencia de la masculinidad en la empresa actual como una problemática que lo es en gran medida por estar esta ideología interiorizada en el inconsciente de los hombres, con lo que esto implica en cuanto a poca visibilidad, dificultades para detectarse, reconocerse y actuar sobre sus limitaciones.

El conocimiento sobre la narrativa masculina y sus consecuencias no es nuevo, pero las acciones para la concienciación social han sido escasas y concretamente en los entornos empresariales prácticamente inexistentes; no en vano continúan siendo unos entornos mayoritariamente masculinos que, a diferencia de los análisis objetivos citados, no suelen reconocer la necesidad de diversificar el personal de la compañía.

No obstante, cada vez más compañías, comenzando por las grandes tecnológicas multinacionales, llevan a cabo políticas internas de diversificación por sexos y étnica, buscando sumar sensibilidades, ampliar la visión, la creatividad y con ello adaptarse ventajosamente a los cambios. Estas grandes empresas 
suelen tener directivos y asesores ampliamente formados con sensibilidad a la influencia de factores identitarios en los negocios, pero no ocurre lo mismo en el caso de pequeñas y medianas compañías.

Esta formación y conocimiento que acumula la gran empresa por sus mayores posibilidades y recursos humanos, sin duda, contribuyen a la consabida competencia desigual entre grandes y pequeños, tendente a la supervivencia de pocas entidades 0 incluso la formación de oligopolios y monopolios, empobreciendo la creatividad, la innovación y la salud de la economía.

La formación en dimensiones identitarias, por tanto, debe ser promovida en pequeñas y medianas empresas (PYMES) desde el ámbito público en el desempeño de su labor como vigilante y promotor de una buena salud de la economía, creando una oferta formativa al alcance de líderes empresariales y no empresariales, difícil de generar desde una pequeña compañía, pero altamente factible desde la administración pública y cuyo costo puede ser trasladado o compartido con los beneficiarios.

La creación de cursos y talleres para la formación de empresarios va dirigida a lograr cambios en directivos activos, pero hay que incidir igualmente en los futuros directivos. La falta de formación en género en los estudios empresariales de la mayoría de las universidades muestra la necesaria actuación en este ámbito tan decisivo. Es común que los planes de estudio económicos planteen un enfoque de género en el estudio de los consumidores o incluso en la gestión de recursos humanos (nivel de paridad), lo que en cambio está ausente es un nivel suficiente de profundización en la materia que muestre la influencia del género en los trabajadores, en la gestión, para poder reducir su influencia negativa fomentando las positivas. Trabajar a este nivel exige educación sobre ideología de género cosa a desarrollar en los estudios empresariales y económicos de las universidades.

El impacto buscado tras la actuación sobre los directivos actuales o futuros se extiende en la economía y la sociedad, abordando la igualdad en la práctica y estimulando los beneficios empresariales de las siguientes maneras, tal y como muestra la experiencia de su aplicación:

- Capacitando a los hombres empresarios, incitando a la reflexión, para superar limitaciones procedentes de la masculinidad ampliando la visión y la actitud de servicio, sin perder las habilidades para competir sino reforzándolas. 
- Promoviendo la inclusión de mujeres en la dirección de la organización, rompiendo el techo de cristal, aprovechando así la diversificación identitaria para incrementar la visión y capacidades de la organización.

- Mejorando la gestión de la compañía en su relación con los clientes y competidores, desde la participación de diversas sensibilidades..

- Fomentando el equipo humano, cohesión y trabajo conjunto, tacto humano dentro de la organización.

- Mejorando el rendimiento y la rentabilidad de la empresa, objetivo último de ésta, y con ello a mayor escala de la economía, con su impacto en la sociedad.

Aunque esta actuación de formación empresarial puede generar un alto impacto, debemos considerar que para realmente efectuar cambios en el conjunto de la sociedad hay que incorporar la educación en género en las etapas educativas de niños y jóvenes.

Esta necesidad otras veces señalada, en la práctica no ha pasado de ser, en el mejor de los casos, una actividad docente puntual dentro de alguna materia humanística del currículo. En numerosos países sí se han logrado avances en cuanto a formación en sexualidad que conlleva una aproximación al género importante, pero más allá existen muchas esferas de experiencia relacionadas con el poder y la violencia, con valores y actitudes, auto-hetero estima, entre otras.

Se han realizado en España, México, Costa Rica y otros muchos países, campañas mediáticas que buscan concienciar sobre acciones que perpetúan dominio entre personas de distinto sexo pero su impacto real resulta insuficiente. Ante lógicas interiorizadas y altamente inconscientes debemos actuar con formación sobre género en las etapas educativas obligatorias. Numerosas autoras y autores han abogado por una educación de género trasversal, es decir, incluida en la planificación de las diversas asignaturas, tratándose de esta manera sus implicaciones concretas en cada área.

Colás y Villaciervos (2007), tras constatar una especial interiorización de los estereotipos de género en hombres jóvenes, detectaron "hombres desorientados, que asumen la igualdad como principio pero no lo practican de forma estable o consciente".

Lo cual indica la necesidad de tener en cuenta la ideología inconsciente, descender al nivel de los valores y narrativas interiorizadas, poniendo en práctica acciones de educación de género eficaces, llevando la educación en género a los 
distintos niveles formativos. El aviso de, Amurrio, et al (2012) sobre las dificultades para afrontar la concienciación sobre la "masculinidad imperante", apunta a ir más allá de las campañas mediáticas, "requiriendo un gran pacto educativo entre todas las estancias socializadoras en las que están insertos nuestros jóvenes".

\section{Conclusiones}

Debemos reconocer la importancia de la conciencia del género en cuanto a constructos ideológicos, sistemas de valores y roles, en los distintos contextos de experiencia social y personal. Precisamente el bajo conocimiento de las múltiples implicaciones de esta dimensión en la vida diaria facilita el que sus consecuencias negativas, en cuanto a reparto de poder o falta de aprovechamiento del potencial humano, no sean abordadas convenientemente.

En el mundo empresarial se ha constatado que las mujeres directivas alcanzan un mayor éxito que la media general y esto ha sido analizado desde el nivel de los sistemas de valores por la coincidencia de los requisitos de la economía actual con las actitudes y roles tradicionales femeninas. Hoy en día la competitividad entre empresas se decide por cualidades como la creatividad, la actitud de servicio al cliente, la escucha y la empatía con los trabajadores y los consumidores, entre otras cuestiones.

Las mujeres la feminidad dan muestras de una mayor flexibilidad y adaptación a los cambios que el constructo de género masculino, que se caracteriza por una gran rigidez. Hemos reflexionado sobre la naturalización de una masculinidad que impide a muchos hombres reconocerla, reflexionar, actuar con mayor libertad y capacidad de adaptación al entorno.

Esto nos lleva a hacer propuestas de formación específica en género para líderes, especialmente del entorno de la pequeña y mediana empresa. Paralelamente, es necesario introducir en los estudios universitarios económicos y empresariales una asignatura de género que profundice suficientemente en esta dimensión, formando convenientemente futuros líderes. Mejoras en cuanto a rentabilidad empresarial, organización interna y el bienestar general están relacionadas con esta aproximación.

Es necesario bajar al nivel de los valores y la narrativa identitaria interiorizada, permitiendo la reflexión personal y así ganar cuotas de libertad y capacidad de actuación. Cursos de masculinidad y género dirigidos a empresarios pueden abordar sus limitaciones personales, al tiempo que contribuir a romper el techo 
de cristal, pero para lograr cambios sociales consistentes y permanentes hay que recordar una vez más la incorporación de la educación en género en el sistema educativo, en niños y adolescentes de ambos sexos.

Concienciar es sacar la ideología a la superficie además de señalar actos concretos, visibilizarla para poder reflexionar sobre si sus valores y narrativa son los adecuados para cada uno y la sociedad. Recursos humanos no escasean para esta labor docente en la empresa, la universidad y la escuela, teniendo en cuenta la cantidad de antropólogos sociales, sociólogos y psicólogos que no encuentran suficientes oportunidades laborales en la economía y sociedad actual.

\section{Referencias}

Adams, R. \& Ferreira, D. (2009). Women in the boardroom and their impact on governance and performance. Journal of Financial Economics 94, 291-309.

Adams, R. \& Ferreira, D. (2007). A theory of friendly boards. Journal of Finance 62, 217-250. Amurrio, M.; Larrinaga, A.; Usategui, E. y Del Valle, A. (2012). Los estereotipos de género en jóvenes y adolescentes. Ponencia en XVII congreso de estudios vascos. Donostia.

Bauman, Z. (2007). Vida de consumo. Madrid: Fondo de Cultura Económica

Eagly, A. y Carli, L. (2007). Women and the labyrinth of leadership. Harvard Business Review. Septiembre, 85(9), 62-71, 146.

Eagly, A. y Carli, L. (2003). The female leadership advantage: an evaluation of the evidence. The leadership Quaterly, 14, 807-834.

Blanco, J. (2012). La masculinidad como factor de riesgo: una etnografía de la invisibilidad. Sevilla, Universidad Pablo de Olavide.

Carr-Ruffino, N. (1991). Us women: breaking through the glass ceiling. Women in Management Review, 6(5).

Diekman, A. y Eagly, A. (2000). Stereotypes as dynamic constructs: Women and men of the past, present, and future. Personality and Social Psychology Bulletin, 26, 1171-1188.

García, M. (2009). La responsabilidad social corporativa y el estilo de dirección femenino. Sección Artículos, Revista Capital Humano, 229, 52.

Gimenez, G. (2009). Identidades sociales. México: Consejo Nacional para la Cultura y las Artes/Instituto Mexicano de Cultura.

Guasch, O. (2003). Ancianos, guerreros, efebos y afeminados: tipos ideales de masculinidad. Valcuende del Río y Blanco. (coords.). Hombres. La construcción cultural de las masculinidades. Madrid: Talasa.

Helgesen, S. (1990). The female advantage: Women's ways of leadership. New York: Double day Currency.

Colás, P. y Villaciervos, P. (2007). La interiorización de los estereotipos de género en jóvenes y adolescentes. Revista de investigación educativa, 25(1), 35-58. 
Colin, M. (2003). New gender gap. Revista businessweek.

Demartini, J. (s.f.) valores para triunfar en los negocios, en www.DrDeMartini.com

Dezso, C. y Gadiss, D. (2012). Does female representation in top management improve firm performance? Strategic Management Journal 33(9), 1072-1089.

Kaufman, M. (1995). Los hombres, el feminismo y las experiencias contradictorias del poder entre los hombres. En: Arango, Luz; León, Magdalena y Viveros, Mará (compiladoras). Género e Identidad. Ensayos sobre lo femenino y lo masculino. Santa Fe de Bogotá: Uniandes.

Kimmel, M. (1997). Homofobia, temor, vergüenza y silencio en la identidad masculina. 49-62. Valdés, Teresa y José Olivarría. (coords). Masculinidades, poder y crisis. Santiago de Chile: Ediciones de las Mujeres.

Ortner, S. (1974). Is Female to Male as Nature is to Culture? Anthropological Theory. 402-413.

Ramírez, E. (2007). Etnicidad, identidad y migraciones. Teorías, conceptos y experiencias. Madrid, Editorial Universitaria Ramón Areces.

Rosener, J. (1995). America's competitive secret: Utilizing women as management strategy. Oxford University Press.

Valcuende, J.M. y Blanco, J. (2015). Hombres y masculinidad ¿un cambio de modelo? Maskana, 6(1), 1-7.

Wechsler, P. (3/3/2015): women led companies perform three times better than the SP500, revista Fortune.

Zuccarino, M. (2012) Modelos estadounidense-fordista y japonés-toyotista: ¿Dos formas de organización productiva contrapuestas? Revista región y sociedad 7(21), 197-215. 